\title{
UM RACCONTO DI GADDA: COMPAGNI DI PRIGIONIA QUARTO ARTICOLO DI GUERRA DELLA PRIMA PARTE DI IL CASTELLO DI UDINE
}

Julia Marchetti Polinêsio

Con Il Castelo di Uline, pubblicato nel 1934 da Solaria, Firenze, lo scrittore Carlo Emilio Gadda vince il premio "Bagutta" e inizia un momento decisivo nella sua arte. Oltre ad essere un'opera importante per capire l'animo gaddiano, vi si trovano già delineate le caratteristiche di linguaggio e di stile che faranno poi di Gadda uno degli scrittori più discussi del suo tempo. Il libro si divide in tre parti: la prima, Il Castello di Udine, composta da cinque articoli di guerra, è come una continuazione del Giornale di guerra e di prigionia che, sebbene pubblicato per la prima volta nel 1955 (Sansoni, Firenze), fu scritto fra gli anni 1916/19, durante la prima guerra mondiale. Ne "Il Castello di Udine" troviamo però un Gadda più maturo e più cosciente delle sue poss bilità artistiche, che già conduce il discorso soggettivo interno verso il nucleo di un tema fondamentale nella sua opera: la meschinità della vita e delle cose.

Nella seconda parte del libro, "La crociera mediterranea", Gadda, visitando Napoli, la Costa Amalfitana, la S:cilia, la Tripolitania e Rodi, segue l'itinerario classico del turismo borghese; caricaturizza il tono pomposo che hanno in genere queste forme di racconto, con un misto di entusiasmo ed ironia e col senso sempre presente della meschinità delle cose.

La terza parte, "Polemiche e pace" - la parola "polemica" é qui usata nel senso ampio di qualsiasi situazione di sforzo o conflitto, alla quale si contrappone, come alternativa, il termine "pace" - è con tituita da prose diverse, unite dal nucleo centrale della concezione della vita nel suo aspetto globale e assoluto.

Comincia con questo libro il tipico "pastiche" gaddiano, che consiste nell'impiego dei più diversi elementi linguistici - termini arcaici, elementi colti e popolari, deformazioni lessicali e sintattiche, termini tecnici, dialettali, stranieri - in impasto coeso e perfettamente amalgamato. 
Ne Il Castello di Udine Gadda usa il procedimento, già impìegato nella letteratura classica, di sdoppiare il foco narrativo incombendo un commentatore immaginario - il Dott. Feo Averrois - di schiarire certi passaggi attraverso abbondanti e dettagliate note in margine. Questo commentatore fa precedere il testo di Gadda di una (volutamente) pedan te "Sinossi delle abbreviazioni usate annotando", il che costi_ tuisce, sebbene in modo molto più tenue, un terzo foco narrativo. Le note del commentatore sono una variazione di Gadda su sé stesso, un metalinguaggio, e danno all'opera una struttura peculiare. Con questo procedimento Gadda non cerca soltanto di captare la realtà con meticolosa precisione, ma ci dimostra, come lo confermano le abbondanti note di altri suoi scritti, quanto sia presente in lui la coscienza dell'importanza del fatto linguistico e l'ansia, mai completamente soddisfatta, della ricerca dell'assoluto.

Il commentatore immaginario, Dott. Feo Averrois, apre cosí il suo discorso, dando inizio al libro:

"Gli editori di Solaria mi hanno commesso d'annotare gli scritti del Gadda (C.E.) raccolti nel presente volume al titolo

\section{IL CASTELLO DI UDINE}

Poi, con manierismo in cui è evidente l'ironia continua:

"( ) Mi fa prossimo al Gadda (C.E.) un'antica dimestichezza: cosí la mia traduzione serà da poter essere considerata autorevole e valida, quanto consente, almeno, l'ambiguo de' di lui modi e processi."

Lo stile di questa introduzione è classico e scolastico, a scopo evidentemente ironico, cosí come di tradizione scolastica è il procedimento di citare l'autore in studio con l'appellativo "ll Nostro" (Ns)

Il racconto che analizzeremo in seguito è il quarto della prima parte, intitolato "Compagni di prigionia" $\vec{E}$, come lo indica il titolo, un ricordo dei momenti passati in prigionia, durante la prima guerra mondiale, presso i tedeschi. Nel 1917, dopo due anni al fronte, Gadda, fatto prigioniero, fu inviato dapprima a Rastatt, in Austria, e trasferito dopo a Celle, nell'Hannover. Questa esperienza amara, che gli ha impedito di partecipare alla guerra, sentita da lui con tutta l'anima, gli ha lasciato una profonda marca e il rammarico perdurò sino alla fine della vita, fatto quasi complesso di colpa per non aver potuto compiere verso la Patria il suo dovere. 
In Il Casteilo di Udine Gadda impi ga già, come abbiamo accennato, il caratteristico impasto linguistico detto "pastiche", ma in "Compagni di prigionia" le deformazioni sono relat.vamente poche e prevale, in una struttura classica, una sapiente elaborazione della lingua senza le deformazioni che si noteranno in altre opere. Possiamo cosí osservare come l'autore ricorre al "pastiche" non come a un art ficio stilistico, cioè, non a vuoto, cosa di cui fu accusato in principio, ma soltanto quando lo richiede la necessità espressiva. "Mi studio di evitare ogni slittamento verso innovazioni meramente narcisistiche. Alla qual bisogna può sovvenire l'ironia, l'auto-ironia. Un tono teso di qualità narcisistica l'ho in uggia, se pure vi possa essere incorso nolente, per difetto d'inibizione estetica: o morale. Per inconsideratezza pecchiamo" (1)

La disgregazione e la riorganizzazione dei material: linguistici devono provenire, secondo Gadda, non da una vana ricerca di originalitá ma da un più profonda necessitá interna. La prosa di "Compagni di prigionia" è anche rappresentativa del Gadda lirico, il quale arriva in certe pagine a momenti di altissima poesia.

Si notano nel racconto tre parti distinte. La prima è descrittiva; attraverso le descrizioni di persone, di situazioni e oggetti si intuisce lo stato d'animo dell'autore, la psicologia dei personaggi e le sofferenze íro. La seconda è un intermezzo lirico. Comincia quando il protagonista, che è lo stesso Gadda, si fa leggere da un compagno le poesie scritte n carcere, e comprende tutto questo episodio. Il poeta è Ugo Betti (1892-1953) che, con Bonaventura Tecchi (1896-1968), fu compagno di Gadda in prigionia; l'amicizia fra i tre scrittori continuò poi per mo'to tempo. Tra questo intermezzo e la parte finale vi è, nella prima edizione, una separazione, non mantenuta in quella di Einaudi. La terza parte del racconto torna a rievocare ricordi di prigion:a e l'au tore la chiude con un disperato desiderio di fuga, di partecipazione, di azione. E importante osservare che quasi tutto il racconto è scritto all'imperfetto. L'azione si fa cosí più imprecisa, p ù vaga nel tempo, non finita, come in una specie di continuità atemporale. Entra il passato remoto soltanto nella seconda parte, quando l'autore, descrivendo il sentimento causatogli dalla lettura delle liriche, lo fissa in un tempo preciso com? per metterlo in rilievo.

Per analizzare il racconto adotteremo il metodo di trancrivere $\mathrm{i}$ brani di cui si vogliono commen are termini o caratteristiche espressive. Questi saranno, ognuno a sua volta, anal zzati dopo la trascrizione de] brano. L'edizione da noi consultata è quella di Einaudi, Torino, 1971.

(1) - Gadda, C.E., "Come lavore" in I viaggi la morte, Milano, Garzanti, 1958, p 21 
Confrontata con la prima, del 1934, troviamo alcune alterazioni che a mano a mano commenteremo. Le note del Dott. Feo Averrois, mentre nella prima edizione vengono in margine e sono 16 invece di 13 , in quella di Einaudi sono raggruppate alla fine di ogni capitolo.

L'inizio del racconto fissa un momento della vita dei prigionieri nella loro baracca, momento che si intuisce uguale in ogni giorno od ora:

"Rivedo la baracca numero quindici, la luce sistematica d'un giorno eguale: e $\mathrm{i}$ compagni scrivere o studiare l'inglese o rammendar panni: o rimestare le loro polte e i tenui imbratti delle lor salse sulla piccola cucina di ghisa." (p. 55).

E comune in Gadda il procedimento di trasferire le qualità dal soggetto all'oggetto. Il modo di viver dei prigionieri, il "sistema", è trasferito alla luce - la luce sistematica - , cosi come eguale non e il giorno, ma la vita di prigione. Questo espediente stilistico, oltre a dare maggior forza al messaggio, permette di coinvolgere le cose in un sistema globale. Troviamo qui, in una sola frase, due metafore: luce sistematica - giorno eguale. Il loro accoppiamento rafforza l'idea di monotonia. I verbi all'infinito - studiare, rammendare, rimestare danno l'idea di un azione che continua atemporalmente, che monotonamente si prolunga.

"Salvatore, siciliano-milanese, calvo come una palla da biliardo e un poco sbilenco, cuocheggiava a tutt'andare dalla mattina alla sera istottendo di tanto in tanto qualcheduno, senza levar gli occhi di dosso al mestolante suo mestolo" (p. 55)

La preoccupazione per il cibo, l'ingegno con cui s'impegnano i prigionieri ad ingannare la fame è tema costante di tutto il Giornale di guerra e di prigionia, e in questo racconto ritorna insistentemente. Lo cominciano a vedere nella frase: "cuocheggiava a tuttandare dalla mattina alla sera", in cui si distacca l'efficacissimo verbo cuocheggiane. " 'Sfottendo' di tanto in tanto qualcheduno": il verbo popolare sfottere, oggi molto in uso, è messo da Gadda fra virgolette perché meno usato e più grossolano quarant'anni fa.

Nell'espressione mestolante suo mestolo (si osservi quanti termini nuovi crea l'autore), il participio presente pleonastico rafforza enormemente il messaggio. Messo prima del soggetto attrae su di sé la carica semantica della frase e l'azione si proietta in primo piano, si fa quasi forma visiva.

“ 'Pierino', come gravato della sonnolenza- polenta d'un eterno dopopranzo (l'unica cosa che mancava era la gialla cosa in sé) 
studiava eternamente il tedesco, ziehen, zug, gezogen, tirato fuor di pagina a ripensare nostalgico la sua Valcamonica". (p.55)

La parola-macedonia sonnolenza-polenta, oltre alla forte carica semantica impressa al messaggio, dà, con la rima interna, un ritmo ondulante alla frase. Osserviamo che continua l'ossessione del cibo: Pierino, non potendo masticare la polenta - "l'unica cosa che mancava era la gialla cosa in sé" - mastica le parole tedesche - ziehen, zug, gezogen - di cui le sillabe in en ripetono il ritmo ondulante iniziato sopra, e il significato - tirare, tirò, tirato - suggerisce come una fuga del personaggio verso la nostalgia della sua valle. L'espressione la gialla cosa in sé, come osserva il Dott. Averrois nella nota 1 del suo commento, è suggerita a Gadda da Kant, poiché das din an sich (la cosa in sé) è una proposizione basica della sua Analitica. "L'oro è giallo, osserva Averrois, "costituisce proposizione esemplare per i giudizi analitici nella Analitica Kantiana" Nella prima edizione questa nota ha una parte che è stata soppressa in quella di Einaudi: "( $\mathrm{J}$. Kant-Kritik der reinen Vernunft - Analitik-Analytische u. Synthetiche Urtiele). Il Ns. non usa queste parole con animo derisorio, ma quasi ad introdurre d'un subito il lettore nel clima germanico, con rifarsi per accenni a uno de' significativi momenti del mondo germanico. Cosí la luce sistemantica'..." Come vediamo, in questa nota veniva spiegata anche la ragione dell'espressione luce sistemstica commentata da noi sopra: è impiegata per connotare il "sistema" della prigione e della vita germanica.

"Difatti m'avvicinavo alla nicchia d'ombra e di malinconia, verso l'uscita della baracca; dove ero sicuro di trovarlo, trasognato e imbronciato, semisdraiato sul suo giaciglio, con il puzzle del Sauer-Ferrari davanti." (p. 55)

Troviamo qui un altro procedimento comunissimo in Gadda: i termini qualificanti sono sostantivi e non aggettivi. Nicchia d'ombra $e$ di malinconia, per "nicchia ombrosa e malinconica", è molto più espressivo; le qualità, sostantivate, acquistano caratteristiche più forti e l'immagine si fa plastica.

"Con il puzzle del Sauer-Ferrari": puzzle, ser "gioco enimmistico", era già entrato in uso corrente nella lingua italiana. Nella nota 2 il commentatore fa una caricatura di certe grammatiche (in questo caso quella di Sauer-Ferrari) con una frase che è un esempio del nonsenso, della non corrisponza del metodo d:dattico con la realtà: "Otto Sauer e Ottorino Ferrari regalarono congiuntamente alla Umanità una cele- 
bre grammatica; e un non meno celebre metodo: "La cameriera della duchessa ha dimenticato il temperino di tua zia sullo scrittoio del governatore, ma il cocchiere del conte ha lustrato gli stivali dell'arciduca con la spazzola del suo bisnonno" L'ironia di Gadda è sempre presente, lucida, mordace.

"Enzo, mio compagno e socio, stava già cucinando a sua volta: all'udirmi cosí sciocco sostava un attimo, scoteva il capo come si fa d'un inguaribile e, dopo ruminatoci sopra un bel po', quand'io credevo che pensasse soltanto alla pentola e arrivavo tranquillo come un trelire (3), ecco una scarica a brucia pelo: mi latrava ingiurie bergamasche a pieno viso con la veemenza terribile d'un orator sacro, accomunando me e qualche altra dignità della celeste piramide nell'emboitement delle sue spaventevoli ipotiposi. ( ) "Pari un bambino" mi diceva: e con nuovi improperi, citando San Paolo, mi esortava a rifare il "letto" e a "disfarmi" (4) la barba." p. 56)

La ripetizione insistente dell'atto di cucinare, o di oggetti relativi alla cucina, indica l'insistenza della loro fame. Presente in tutto il testo, ci dà l'idea di un'azione continua, come se i prigionieri non si occupassero che di questo. E la trasposizione della qualità del soggetto all'oggetto, fatta però qui in forma implicita; cioè, la fame dei prigionieri non è espressa direttamente, ma attraverso gli atti praticati e gli oggetti citati.

"Tranquillo come un trelire": è questo un termine italianizzato del dialetto lombardo, spiegato da Averrois nella nota 3, perché incomprensibile a chi non conosce questo dialetto: "La frase classica è: "pacific come on trelira', e dicesi di chi cammina placidamente"

"Nell'emboitement": Gadda impiega parole straniere senza virgolette o alcun segno che le metta in rilievo, come appartenenti al nostro lessico. Oggi questo procedimento è diffuso, ma non lo era al tempo in cui il libro fu scritto.

"Mi esortava a rifare il "letto" e a "disfarmi" la barba: letto è fra virgolette per ironizzare il precario giaciglio dei prigionieri. Il verbo disfare riferente a barba è del dialetto lombardo, e viene spiegato nella nota 4: "Desfaà la barba = far la barba: t. lomb."

"Una nuvola di polvere e di farinetta si spandeva tutto attorno, (come quando si battono i più doviziosi tappeti), sugli approntamenti culinari degli altri e sulle vettovaglie artisticamente disposte, hors-d'oeuvre pieni di fede, di speranza e di dignità." (p. 66) 
Vediamo che appare sempre, tema dominante, la preoccupazione per il cibo; la fede, Ja speranza e la dignità attribuiti agli horsd'oeuvre sono una magistrale trasposizione sull'oggetto delle qualità morali di chi li prepara.

"S'era stabilita fra noi, nonostante mille bizze e litigi, una fraterna vita. "Via", mi diceva dolcemente Enzo, passando dall'ira alla pietà e dal bergamasco al toscano, "Fatti la barba! Non vedi cosa sembri?" (. ) Mi apprestavo alla barba, commosso, sospirando a mia volta, con tutta la drammaticità di cui sono capace. Tutti se ne accorgevano subito: fiocchi di spuma volavano alti sopra i pentolini e la cucina e mannagge cupe (queste qui di mia propria fabbrica) offendevano il pio sentire d'alcuni, e poi di tutti. Si volgevano esterefatti, poi impensieriti, poi ammirati. (...) Al primo sangue, tutti mi si facevano intorno: "Ma che bravo!" "Bravo Gadda, casí mi piaci" "Cosí deve essere un ufficiale italiano!" "L'esempio viene dai migliori" "Sarai proposto per l'avanzamento!" "Vedrai! Ti daranno la medaglia d'argento, stavolta, invece del bronzino che t'han dato!" Ferito nella mia spasimante vanità, li minacciavo furibondo, il sangue vividamente gocciava nel collo, faceva come un collare di porpora nella sciocca ironia dello specchio." (p. 57/58)

Troviamo inserito nel testo il temine d:alettale mannagge. È una nota imprecazione del dialeto napoletano, qui sostantivata, impiegata nella frase come appartenente al lessico italiano. La fusione di elementi linguistici eterogenei, che costituisce il "pastiche", appare in quest'opera ancora parsimoniosamente, e raggiugerà il suo punto massimo nel romanzo Quer pasticciaccio brutto de Via Merulana. 'Il 'pastiche' di Gadda", osserva il Devoto, "ha caratteri suoi particolari che lo distinguono pur nella diffusa tendenza allo sperimentare linguistico che caratterizza i nostri anni. Il problema linguistico di Gadda non si racchiude nel problema del rapporto fra lingua e dialetto, ma investe tutti gli aspetti e tutte le possibilità della lingua." (2)

"Il sangue vividamente gocciava nel collo, faceva come un collare di porpora nella sciocca ironia dello specchio" la situazione tragicomica dei prigionieri, che con scherzi e risate tentano di mascherare la loro miseria, è trasferita allo specchio, il quale scioccamente e ironicamente riflette tutto. Un'altra volta troviamo lo stilema già osservato avanti: un sostantivo impiegato al posto di un aggettivo, come termine qualificante. L'aggettivo qualificativo ironico è sostituito dal

(2) - Giacomo Devoto e Maria Luisa Altieri, La lingua italiana Storia e problemi attuali, ERI, Torino, 1968. 
sostantivo astratto ironia. Qui lo stilema si fa ancor piu complesso, perché il sostantivo ironia è, a sua volta, specificato da un altro aggettivo. Cosí, ironia dello specchio sta per "specchio ironico", e sciocca ironia dello specchio sta per "specchio sciocco e ironico" Roscioni osserva che questi procedimenti stilisici hanno la funzione di portare in primo piano le qualità delle cose, e di "sottolineare che è soprattutto grazie a queste proprietà che esse vengono percepite" (3)

"Serio, alto, pallido, Betti (5) rientrava, 'subtilis atque elegans atque disertus puer' Si levava il cappotto, riponeva qualcosa, libro o fascicolo, sotto il suo 'materasso'. Non si sapeva che cosa. Al vedemi cosí concitato, mi si avvicinava con una impercettibile piega nei labbri, segno appolineo di crudeltà, come un dio che cogliesse, in peccato d'irriverenza, il mortale. Io lo guardavo in tralice venir avanti, impegnato in quello zabaglione di sapone e di sangue, con mille ferri ginecologici sopra il "letto", vani a darmi la pace barbina. Un tetro malumore mi prendeva tutto, muscoli e nervi. (...) Insaponato una terza volta, grugnivo, animale selvatico e malinconico, ferito e ingabbiato.

Tecchi (6) rientrava, con quella sua andatura tremenda di persona che s'è proposta camminare a tutti $\mathrm{i}$ costi, contro a tutti. Riponeva i suoi libri, salutava breve e secco, e come distratto ne' suoi avventurosi pensieri, in po' stanco dallo studiare.

"Come va Gaddone? Hai lavorato?" mi chiedeva. togliendosi il pince-nez. (p. 58)

Per riferisi al colto ed elegante Betti, Gadda impiega parole latine. In Giornale di guerra e di prigionia ne parla con ammirazione: "Classe '92, studente in legge; ha grandi conoscenze di letteratura e Tecchi lo stima il più intelligente di noi. E pure un buon ginnasta, ottimo corridore e saltatore, ottimo alla sbarra e alle parallele. Ciò lo cresce nella mia stima" (4) Nelle note 5 e 6 del commento di Averrois sono specificate le opere e le qualità di Tecchi e Betti. Più avanti, nel racconto (p. 65), Gadda ritorna a parlare con ammirazione di questi suoi due compagni.

"Al vedermi cosí concitato mi avvicinava con un'impercettibile piega nei labbri": Gadda è solito usare al maschile il plurale di "labbro', "lenzuolo", "dito" Ma non sempre. Troviamo anche, alle volte, la

(3) - Gian Carlo Roscioni, La disarmonia prestabilita, Einaudi, Torino, 1969 p. 22.

(4) - Gadda C.E., Giornale di guerra e di prigionia, op. cit., p. 198. 
forma tradizionale (5), il che conferma la nota sua frase: "I doppioni li voglio, tutt:, per mania di possesso e per cupidigia di ricchezze: e voglio anche $\mathrm{i}$ triploni, e i quadruploni. e tutti i sinonimi, usati nelle loro variegate accezioni e sfumature, d'uso corrente, o d'uso raro rarissimo" (6)

"Con mille ferri ginecologici sopra il "letto", vani a darmi la pace barbina": l'aggettivo barbina che qualifica "pace" è invenzione di Gadda, ma pace barbina ha anche il senso recondito di "pace meschina, gretta"

"Insaponato una terza volta grugnivo, animale selvatico e malinconico, ferito e ingabbiato": Gadda si sente ingabb ato non solo per la ragione esplicita della prigionia, ma per la sua predisposizione a soffrire. Egli molto si confessa, pochi scrittori hanno, come lui, tanto pienamente messo a nudo la loro anima. La sua malinconia la espone e la studia in tutte le maniere, e disperatamente cerca di spiegarsene la causa. Nel romanzo La cognizione del dolore la chiama "il male invisibile"

"Tecchi rientrava, con quella sua andatura tremenda di persona che s'è proposta camminare a tutti i costi, contro a tutti": con una pennellata Gadda fa il ritratto morale di Tecchi, col quelle, più che con Betti, si legò di amicizia. (7) Tecchi capí meglio il tormentato scrittore e lo stimava molto, mentre Betti (lo dice Gadda stesso nel Giornale di guerra, ma senza rancore) non seppe comprendere o intuire la sua complessa personalità. Nel nostro racconto, sebbene non sia chiaramente espresso, tut!o ciò lo si percepisce dal modo con cui ambedue lo trattano: agressivo il Betti, affetuoso e comprensivo l'altro.

"Togliendosi e ripulendosi il pince-nez": questa è l'unica volta in cui Gadda impiega, nel testo, una parola straniera in corsivo; farse perché il termine straniero non è usato per ragione espressiva, ma per necessità lessicale.

"I quattro del gruppo Ernesto (detto il Re dei pacchi) sedevano allora al tavolino quadrato che s'erano potuti combinare, credo con assi divelte dalle spalliere di una latrina, a opera di qualche audace

(5) - I plurali in a (le labra, le dita) Migliorini non li considera di genere neutro, ma plurali femminili di origine neutra, in opposizione ai generi maschili e femminili. La tendenza della lingua è oggi sopprimerli. V Bruno Migliorini, Lingua d'oggi e d'ieri, Sciascia, Roma.

(6) - Gadda C.E., "Lingua letteraria e lingua dell' uso", in I viaggi la morte, op. cit., p. 95.

(7) - A Tecchi Gadda dedicò il Giornale di guerra e di prigionia con le parole: "Ricordando la sua fermezza nei gierni difficili" 
intermidiario; mentre noi mangiavamo que a là, seduti sui giacigli, e chi sulle panche alla tavolaccia comune; con la scodella sulle ginocchia, o con i gomiti e la scodella e la miseria della testa sopra la tavola. I quattro sedevano al tavolino quadrato, come per un gastronomico "poker" da cui fosse escluso il bluff" (p.59)

"Mentre noi mangiavamo ( ) con la scodella sulle ginocchia, o con i gomiti e la scodella e la miseria della testa sopra la tavola": troviamo qui lo stesso stilema commentato sopra, cioè, un sostantivo astratto al posto di un aggettivo qualificativo: miseria della testa, per "testa miserabile" Si osservi come con questo procedimento la qualità dell'oggetto, sostantivata, spicca in primo piano e diviene il centro de! messaggio.

"I quattro sedevano al tavolino quadrato, come per un gastronomico "poker" da cui fosse escluso li bluff": le due parole inglesi sono impiegate una fra virgolette e l'altra no. La ragione sta nella necessità di risaltare il termine poker, che ha qui una connotazione differente da "gioco" Gadda mette le parole straniere in rilievo soltanto quando ha una precisa ragione espressiva.

"La signorile corpulenza di Ernesto, più giovane e più equilibrato di me, specie $\mathrm{n}$ funzione di prigioniero, mi imponeva un senso di simpatia e di rispetto. Con lui non osavo scherzare. Egli leggeva molto e aveva gusti di persona finissima, educata e colta. Apparteneva a quella ricca borghesia milanese, che, nonostante i miei giambi, è stata una realtà, delle più attive e più salde, nella vita economica e morale della patria; parlo della vecchia classe, non di quella cosí variopinta, venuta su all'ultimo, a ministrar case luride e riscuoter fitti arpagonici (7)." (p. 59)

Parlando bene della buona borghesia milanesa Gadda si contraddice, perché neanche per questa ebbe grande simpatia. In vari suoi scritti vi è una satira violenta anche contro questa clesse. La contrad. dizione è sub to corretta nella nota 7 , dove Averrois dice: "Altrove poi deride e stramaledice anche quella"

"Fitti arpagonici": nella prima edizione leggiamo fitti giudaici. L'alterazione è coeren'e, perché mai Gadda dimostrò la minima ostilità verso il popolo ebraico.

"Una tradizione di signorile gentilezza, di misura e di ritenuto gusto era dovuta venir defluendo in quella sua dignitá sistemata, di ragazzo già uomo" (p. 59) 
Il testo si riferisce ancora a Ernesto. La sequenza dei verbi era dovuta venir defluendo - e in special modo il verbo venire seguito dal gerundio, ndicano continuità di azione. La continuità è qui anteriore al tempo presente, data dal trapassato prossimo; la sequenza dei verbi rallenta il ritmo della frase, perché lento è anche nel tempo il processo evolutivo delle qualità morali del personaggio.

"Sazio, mi buttavo sul giaciglio. Ma Betti arrivava; e la sua natura apollinea, dopo colazione, si traduceva a volte nella calma fermissima dell'ufficiale d'alto ritegno, pàllido e senza sorriso; artiglieria da campagna: a volte si spampanava nella truculenza allegra del barbiere-ginnasta in vena d'esibire i muscoli: gargarizzava un'aria, un accenno. Lodava il Regio; e la intelligente città di Camerino, sua patria, e la intelligente città di Parma, sua patria seconda.

Allora, levatomi a seder sul sacco, cominciavo a parlar male di Verdi. E lui per rivalsa usciva in pazzi sarcasmi contro i milanesi, e ferocemente se la prendeva con Giovanni Bertacchi: "Siete gli adoratori di Bertacchi!" Gli tremavano i labbri, impallidiva: "Gadda, a casa sua, sul suo tavolo, cià un Bertacchi alto cosí, capite? cià speso cinquanta lire; si sazia di Bertacchi, combina delle orge di Liriche Umane, balla nudo come i dervisci, come i coribanti, finché cade esausto per terra, completamente imbertacchito.

E andava avanti mezz'ora a descrivere il mio immaginato libro, e l'immaginata scinnide" (p. 60)

"A volte si spampanava nella truculenza allegra del barbiere-ginnasta": spampanarsi, per "vantarsi", è un termine arcaico, poco usato.

Barbiere-ginnast?, parola-mecedonia: barbiere per il canto (dal Barbiere di Siviglia), ginnasta per le qualità atletiche del personaggio. Queste parole create da Gadda collacostamento di termini eterogenei, per formarne un altro di significato differente, sono sempre espressive e sintetiche.

"E ferocemente se la prendeva con Giovanni Bertacchi": G:ovanni Bertacchi fu professore di letteratura italiana all'Università di Padova e autore di poesie di non grande valore, che però Gadda effettivamente amava leggere.

"Gadda, a casa sua, cià un Bertacchi! (....) Cià speso cinquanta lire": Cià è una caratteristica flessione lombarda del verbo avere (da "ci ha"), molto diffusa fra il popolo, usata qui per canzonare i milanesi. 
"Balla nudo come i dervisci, come i coribanti ( ) e andava avanti mezz'ora a descrivere l'immaginata scinnide": osserviamo qui parole ricercate e rare. Di ogni mezzo si vale Gadda per arricchire la lingua. "La lingua dell'uso piccolo-borghese, puntuale, miseramente apodittica, va bene, concedo, è lei pure una lingua; un "modo" dell'essere. Ma non può diventare la legge, l'unica legge. Ripudio un tale obbligo e una sifitta legge"

"Tutti lo seguivano allegri nella sua feroce apologia adversus Mediolanenses; poi di nuovo tiravano in scena la barba. il rasoio, il saccone, la medaglia di bronzo, il fiorito perorare di Enzo, finché Enzo si levava a un tratto fuori dalla grazia di Dio, ad abbaiare contro tutti, violentissimo, travolto dall'ira nelle più vetuperose invettive, in difesa degli alpini di Lecco e di Bergamo: (cari, eroici compagni!) Una clamorosa e generale battaglia di parole e di lazzi, d'ire e di risa, teneva tutta l'aura greve e fumida della baracca; dove s'incrociavano barba e Bertacchi, Verdi e pentolini, Rimbaud e Giuseppe Garibaldi, che si accusava il siciliano di chiamarlo Karbàra, in Sicilia; e Bergamo e Roma, e Caporetto e medaglie d'argento: ed Enrico col vino delli Castelli." (p. 60)

Osserviamo in tutto questo brano trascrito un'altra caratteristica importante dello stile di Gadda: l'enumerazione. L'autore impiega sequenze di sostantivi, verbi, aggettivi, elenchi delle cose più diverse $o$ affini, con lo scopo di riprodurre il disordine e la confusione della vita, e molto spesso anche a fine ironico. Questo procedimento è frequentissimo nella sua opera, dai primi agli ultimi scritti. "La ragione di questo processo sta nel fatto che alle idee di molteplicità e di varietà si associano naturalmente quelle di aleatorietà e di confusione." (9) Qui Gadda si serve dell'enumerazione di cose senza alcun nesso logico fra loro, per esprimere lo stato d'irritabilità e d'angoscia dei prigio. nieri.

"Una clamorosa e generale battaglia di parole e di lazzi, d'ire e di risa, teneva tutta l'aura greve a fumida della baracca": tutto il periodo precedente a questa frase, messo in rilievo dal sostantivo aura del noto verso di Dante - "quell aura senza tempo tinta" (Inf. III-29) - evoca un clima dantesco, riproduce l'idea di un amb:ente infernale, idea rafforzata dalle espressioni e dai termini impiegati: abbaiare, violentissimo, travolto dall'ira, vituperose invettive, battaglia di parole

(8) - Gadda C.E., "Lingua letteraria e lingua dell'use", in I viaggi la morte, op. cit. p. 98

(9) - Gian Carlo Roscioni, La disarmonia prestabilita, op. cit. p. 42. 
$e$ di lazzi, ecc. Unica pausa di dolcezza è l'osservazione accorata, messa tra parentesi, - "cari, eroici compagni" - riferita agli alpini.

"La tentazione degli scacchi era immediata e dolce, più forte d'ogni stanchezza, dissolvitrice d'ogni immediata pena. $\mathrm{Ci}$ impegnavamo a fondo. (. . ) Adesso Anguissola suggeriva a Betti qualche mossa, fumando, fumando, all'impiedi. Nuvole di fumo di toscano da un ghigno sardonico. (.. ) Anguissola, allo scacco matto, rideva, rideva improvviso, una vibrazione satanica; poi osannava alla Divina Giustizia e all'angelica tromba, citava l'Allighieri-Oderisi contro i superbi "Oh, vana gloria dell'umana posse!. "; si vantava d'aver procurato a Ugo la vittoria, come il Desix al Buonaparte, e conchiudeva con il motto gentilizio di sua discendenza: "Anguis sola victoriam fecit" (8). E spiegava, chi non lo sapesse, che $\mathrm{i}$ serpenti in latino son fémmine: e cosí anche la biscia viscontèa, che con tanto onore e tanto ardire e fortuna ai suoi agnati avevan portato contro ai nemici, nella furibonda battaglia .

Serenissimo e simpatico uomo, polemista violento - nemico implacabile dell'Inghilterra - ch'era l'autrice di ogni nostra continentale miseria. Il blocco della Germania non gli andava a fagiólo: i mezzi toscani sí". (p.61)

"Nuvole di fumo di toscano da un ghigno sardonico": è un costrutto nominale efficacissimo, che mette in rilievo l'azione. L'assenza del verbo lo fa più evidente; la frase è fortemente plastica.

"Anguis sola victoriam fecit": la nota 8 spiega questo detto: "L'anguis è la vipera dei melanesi. 'Bastò la vipera a determinare la vittoria'"

La parola "milanesi" è alterata: melanesi, da Mediolano. Gadda ni questo brano rievoca il passato, cosa che fa spesso nei suoi scritti, senza soluzione di continuità. Uno dei temi dominanti della sua filosofia è sentire la vita come un flusso continuo, come un passaggio ininterrotto da una generazione all'altra, una continuità. "La continuità, beninteso, non della vita individuale, ma di un processo di cui la vita individuale non è che l'effimera e necessaria manifestazione" (10)

"E spiegava, chi non lo sapesse. ": troviamo, in questa frase, la soppressione della preposizione $a$ (chi non lo sapesse, per "a chi")

(10) - G.C. Roscioni, La disarmonia prestabilita, op cit. p. 42. 
Questo procedimento corrisponde all'attuale tendenza della lingua di eliminare i legami semantici. (11)

"Il blocco della Germania non gli andava a fagiòlo: i mezzi toscani sí": Gadda fa un gioco di parole col termine fagiolo, e lo spirga nella nota 9: "Del blocco, e della consequente carenza di fagiòli, la Germania si rivaleva 'in primis' sui prigionieri italiani”

"Volevo ad ogni costo sapere che cosa facesse Betti nelle ore di mattina, quando si involava solitario. Lo trovai una volta nella baracca brodaglia, semideserta e greve già della disperazione di alcuni miserabili tutt'intorno la stufa; la circondavano con le mani protese verso il tepore; o erano a palarvi carbone. Seduto alla tavolaccia, sulla panca, nel fondo, Betti scriveva: una lettera, avevo pensato. Ma poi, ripensando alla lettera, rivedevo lui con il volto serio e pur dolcemente intento sul foglio, con il lapis a mezz'aria, come un bel galletto che, levata la zampa, sta per fare un passo di più verso l'amorosa battaglia. Era troppa fatica per una lettera, neanche bambino a Natale avrebbe sostato cosí (10). Forse inseguiva i suoi sogni, forse la rima, gallinella procace, o deludente in circolo" (p. 62)

"Lo trovai ( ) nella baracca-brodaglia": altra espressiva parolamacedonia: questi due sostantivi accoppiati rappresentano il miserabile alloggio dei prigionieri, molto meglio di una perifrasi.

"Ma poi, ripensando alla lettera, rivedo lui ( ) come un bel galletto che, levata la zampa, sta per fare un passo in più verso l'amorosa battaglia": il confronto è molto efficace. Spesso Gadda riccorre al confronto per rappresentare immagini o idee. Si preoccupa sempre dei rapporti fra le cose, di "rimettere alle parole e alle favole un mandato provvisorio", di "incastonare le parole nella necessità del momento" (12)

"Forse inseguiva i sogni, forse la rima, gallinella procace, o deludente in circolo": troviamo nel racconto vari participi presenti, sia mantenendo il senso verbale, come nel caso, sia (più frequentemente) svolgendo una funzione di aggettivo: risorgente speranza ( $\mathrm{p}$. 63), saettanti rondini (p. 64), fuggente paese (p. 66). Bruno Mi-

(11) - G. Devoto e M. Luisa Altieri, in La lingua italiana, op. cit., osservano che questa tendenza, iniziata col futurismo, si diffonde sempre di più, ed ha la funzione di mettere in rilievo il significato dei singoli termini.

(12) - Gadda C.E., "Meditazione breve circa il dire e il fare", in $l$ viaggi la morte, op. cit. p. 38 . 
gliorini osserva che il participio presente tende sempre pì̀ a staccarsi dal verbo per farsi aggettivo. (13) Anche in funzione aggettivale il part cipio presente dà alla frase un'idea di azione, un senso attivo .

"Poi, giorno per giorno, si lasciò convincere"

Comincia qui la seconda parte del racconto, che è tutta lirica. La lettura delle liriche di Betti porta all'autore una malinconica nostalgia della patria e della madre, un'ansia impossibile di libertà. Il tempo verbale, tranne un'introduzione che funge da passaggio, passa dall'imperfetto al passato remoto. Anche il luogo dell'azione cambia: dall'interno della baracca passa all'esterno, "verso i reticolati e la sentinella zoppa, là dove si scorgeva la solitudine tutta della brughiera" Quanto di tristezza c'è in questa solitudine tutta, che si fa molto più completa e più ampia con la posposizione dell'aggettivo indefinito.

"Una mattina che le notizie dell'Italia e dell'Intesa eran buone, traverso ritagli di giornali ch'eran potuti pervenirci nei pacchi, e traverso il giallore dei comunicati lor propri (11), una mattina egli era sereno" (p. 62)

Il complemento di tempo - una mattina - messo all'inizio del periodo e ripetuto sotto, acquista rilievo fra gli altri elementi, risaltando cosí il momento in cui inizia l'azione che serà descritta. "Una mattina che le notizie. ": che per "in cui" è un impiego volutamente incorretto del pronome relativo, ripetuto più avanti in identico caso: "gli anni che i miei fratelli avevano piccole mani" (64)

"Il giallore dei comunicati lor propri": l'aggettivo si fa sostantivo: giallore dei comunicati, per "comunicati gialli" La qualità, fatta soggetto, è quasi personificata.

La nota 11 spiega che lor propri è riferito ai tedeschi e non ai pacchi. I commenti del Dott. Averrois alle volte sembrano superflui: rıcordiamoci che il compito del commentatore è far sembrare meno oscuro "ai più chiari Ingenii d'Italia, il convoluto Eraclito di Via Sim. pliciano" Nello spiegare l'ovvio, come in questa frase di Averrois, c'è una evidente intenzione ironica.

"e forse nel mio stesso animo deveva esservi qualche cosa di più lieto e di più giusto, perché mi condusse verso i reticolati e la sentinella zoppa, (il fucile non era zoppo), là donde si scorgeva 
la solitudine tutta della brughiera e la lontana cimasa delle abetaie. Oh! i tedeschi non imboscavano certi stoccafissi di due metri piantati, ma i feriti e gli zoppi delle loro battaglie: verità è verità $(12)$.

Il vento, che nel marzo ancora levava le spire dell'arena, fiore àrido della brughiera per la mia disperata vergogna, il vento s'era come dimesso: la primavera di là della gabbia fioriva di qualche fiore pallido i regni preclusi. E un alito dolce veniva, come un pensiero, come della mia terra e dalle legioni della Piave. Pregavo! ma sentivo quanto indegna cosa mi fosse. "Ecco", disse Betti; e seguitando camminare lungo i reticolati prese a legger quei versi con che oggi s'inizia il suo libro: "Quando il cielo ritorna sereno.

Nella sua voce spiccata e un poco tremente, come d'un fanciullo che dica con inadeguata voce il suo straziato rimpiangere, vibravano accenti chiari dell'anima: e furono $i$ suoi versi come un conforto, e una risorgente speranza. Ma la nostra speranza era affidata ad altri (13), altri soltanto potevano! Neppure il dolce e tragico iddilio di Ugo ebbe virtú di guarirmi. ( . ) La sentinella zoppa adempiva al suo dovere di là dai reticolati, aveva adempito a tutti, a tutti i doveri! Sullo sfondo lontano del dolore il verde cupo dell'abetaia. Oh! il vento era libero nel cielo alto!

"( ) là donde si scorgeva la solitudine tutta della brughiera e la lontana cimssa delle abetaie": molto spesso Gadda riccorre a simboli come a questo, che è l'immagine, tanto frequente nella sua opera, degli alberi, "simbolo di un pagano, incorrotto e religioso passato, dal quale gli uomini si sono allontanati, preogressivamente degradandosi” (14)

Oh! í tedeschi non imboscavano certi stoccafissi di due metri piantati": nella nota 12 Gadda commenta la sua ammirazione per $\mathrm{i}$ soldati germanici. Anche nel Giornale di guerra si osserva quanto l'autore ammiri la serietà e la disciplina di questo popolo.

"Il vento, che nel marzo ancora levava le spire dell'arena, (.....) il vento s'era come dimesso.": tutto questo periodo riflette un triste desirerio d'evasione. Nell'espressione le spire dell'arena vi è un'evidente allusione al verso di Dante: "come la rena quando a turbo spira" (Inf. III - 30) II soggetto vento viene ripetuto dopo una prima lunga frase e il periodo acquista un più ampio respiro.

(14) - Gian Carlo Roscioni, La disarmonia prestabilita, op. cit. p. 56 
"Ma la nostra speranza era affidata ad altri"s l'autore spiega nella nota 13 il messaggio già chiaro: "“ . altri . '= i combattenti d'Italia" È questa l'ultima nota dell'edizione di Einaudi.

"Sullo sfondo lontano del dolore il verde cupo dell'abetaia": questo costrutto nominale mette in rilievo l'immagine. Un'altra volta appaiono gli alberi come sentinelle del dolore dei reclusi. Nella frase c'è una efficace trasposizione, operata dalla metafora sullo sfondo lontano del dolore. Insieme agli alberi, il dolore si fa sfondo, e cosí i due sostantivi, quello astratto e quello concreto (dolore-alberi), sono messi sullo stesso piano, dandoci ambedue un senso di perennita e di altezza.

"Oh! il vento era libero nel cielo alto!": la suggestione di libertà data dalla natura, libera oltre i reticolati, predispone l'animo dell'autore a una malinconia più dolce, mentre era rabbia e rivolta nell'innell'interno della baracca .

"E fu, allora, per me, lo strazio della rievocazione: una musicalità perduta e nostalgica mi richiamò la mia casa di Brian$\mathrm{za}$, i lunghi pomeriggi e le sere del luglio trascorsi correndo, giocando, amici delle saettanti rondini, gli anni che i miei fratelli avevano piccole mani, grandi, purissimi occhi: gemme che coprivo di baci.

Ed è nella sera come un gridío

di bambini

che dicono: addio! addio!

Non so perché questi versi li associavo dolosamente, forse una tragica e oscura prescienza, all'immagine d'un fanciullo, ch'era oggi soldato d'Italia, che non dovevo più rivedere sulla terra!

Altri mi ricondussero, con desolato strazio, alla mamma, e di quelli germinati profondi, non credo parerò facile o temerario all'esegesi, se dirò la mia idea, che soltanto una licenza di guerra abbia potuto dar vita al secreto lor gérmine." (p. 64)

L'autore evoca qui con dolcezza e nostalgia la villa di Longone, in Brianza, costruita dal padre nel 1899, che fu motivo, per lui, di tante sofferenze. Tema importante di La cognizione del doloré, la villa fu una delle cause del dissidio con la madre, che voleva conservarla malgrado la loro precaria situazione finanziaria.

"Ed è nella sera come un gridío

di bambini

che dicono: addio, addio!" 
Questi versi di Betti portano a Gadda il ricordo del fratello Enrico, all epoca combattente nell'aviazione, che, morto nel 1919, non avrebbe più rivisto. Questa perdita fu per lui causa di perenne dolore.

"Altri mi ricondussero, con desolato strazio, alla mamma": qui l'autore ha un pensiero dolce e nostalgico per la madre; i loro rapporti furono, però, molto complessi. Gadda ebbe, verso la madre, sentimenti ambivalenti fra amore e odio; questo rapporto doloroso e difficile fu più tardi analizzato con coraggiosa introspezione nel romanzo, considerato il suo capolavoro, La cognizione del dolore. In un saggio, "Psicanalisi e letterautura", egli studia i rapporti tra genitori e figli e la loro influenza sulla letteratura. "I rapporti tra genitori e figli non sono sempre, non sono per tutti cosí idillici, come certa edificazione semplificante vorrebbe darci a pur bere: e non sono tali perché il sentimento, il sentimento vero, non si fonda sulla rettorica dei buoni sentimenti, ma su quell'aggrovigliato complesso di cause e concause che Freud ha tentato appunto di sgrovigliare" (15) Quest'ultima frase "quell'aggrovigliato complesso di cause e concause" - contiene il nucleo di tutta la filosofia gaddiana, che serà poi approfondito e mag stralmente sviluppato nel suo grande romanzo Quer pasticciaccio brutto de Via Merulana.

La terza parte del racconto è una rievocazione dei compagni di prigionia e vi spicca, viva, la figura di Tecchi. Il tempo verbale è di nuovo l'imperfetto, perché l'andamento ritorna atemporalmente indefinito come nella prima.

"Tecchi mi fu amico generoso e sensibilissimo per tutto il tempo che durò la prigionia (e poi oltre), fino alle ardenti speranze della vigilia e alle prorompenti certezze della vittoria. ( . ) Natura nobilissima di giovane, tre volte ferito, divideva con me il mio rabbioso militarismo e guerrismo, con Betti e con gli altri compagni l'alto spirito d'italianità, che li faceva cosí puri nella mia idea. ( ) Anche con Tecchi solevo camminare giú e su dentro la gran gabbia del Lager. ( ) Qualche volta a noi si accompagnava anche Ottone, curioso d'ogni studio e pensiero, sanissimo e forte giovane. (. .) Se camminavamo noi due soli, allora mi parlava con ammirato amore di suo padre, ingegnere de' piú attivi e provetti della regione. Il lungo lavoro del padre, nove figli, lunghi anni d'amore: tutto, tutto diceva. Perché al solito la mia irrefrenabile necessità deambulatoria, decine di chilometri giú e su per il campo, come il puma in gabbia, malinconica belva,

(15) - Gadda Carlo Emilio, "Psicanalisi e letteratura", in I viaggi la morte, op. cit. p. 47 
riusciva a far fronte alla chiacchierata di Tecchi, tutta angoli, e soste e balzi impetuosi, e poi a quella di Ottone, calda e pacata d'una gran sanità umana, filiale e fraterna, ed emilianamente rotonda.

Cosí la sera scendeva, nuvole basse trasvolavano sopra i fari del campo, rotonde e livide: quasi a lacerarsi nel filo spinato: povere ombre uscivano con una scodella dalle baracche, verso la distribuzione del mangiare, ravvolte ne' tetri mantelli. Le finestrette delle baracche si illuminavano, e noi camminare e camminare. Ottone solo parlava, poi mi lasciava, alla mia desolata inutilità. Pensavo allora, sul Grappa, le scheggie pazze della battaglia, i contrassalti furenti: in una forma di delirio sognavo, vedevo, volevo, vedere! Veder le granate a smontare pezzo per pezzo le corone delle trincee sopra le quote bruciate e i compagni andare, sapendo, sul monte! Avevano tre limoni, baionetta alla mano, magnifiche fòlgori davano a loro il lor senso e quasi una transumana vita: e voleto imitarli e seguirli, dal soglio delle opere prese altri monti vedere, altre schiere avverse, altro fuggente paese. Fuggenti sopra la gabbia non erano che nuvole perse, tetre, nere. Camminavo e camminavo, fagotto di cenci, sulla strada buia dell'eternità" (p. 66)

Attraverso la caratterizzazione del modo di parlare dei personaggi sono rappresentati i loro caratteri differenti. La chiacchierata di Tecchi, tutta angoli e soste e balzi impetuosi", indica il suo carattere nervoso e impulsivo, in opposizone a quello calmo di Ottone, rappresentato della sua maniera di parlare "calda e pacata d'una gran sanità umana, filiale e fraterna" Questa frase, composta con la predominanza della vocale $a$, ha un ritmo lento e calmo, in opposizione a quella che si riferisce a Tecchi, il cui ritmo rapido e balzante - "tutta angoli e soste e balzi impetuosi" - corrisponde al carattere del perso_ naggio.

"Emilianamente rotonda": l'espressione serve ancora per caratterizzare Ottone. L'aggettivo rotondo è usato qui in modo tutto nuovo: connota cosa piana, liscia, senza agoli.

"Cosí la sera scendeva ( ) e noi camminare e camminare": la tristezza della sera che scende, le nuvole basse, le tristi ombre dei prigionieri portano l'autore a un disperato desiderio di fuga. I due verbi allinfinito - camminare e camminare - , la soppressione della preposizione, creano un'impressione di azione continua, senza fine e senza speranza. Lo stesso verbo, ugualmente ripetutto, è ripreso nell'ultima frase del racconto:" camminavo e camminavo, fagotto di cenci, sulla strada buia dell'eternità" L'inutile camminare dei prigionieri si fa una marcia infinita nel tempo. 
"Pensavo allora, sul Grappa, le schegge pazze della battaglia. ": il desiderio di azione passa, vibrante, alla parola. L'intensità del desiderio - "in una forma di delirio sognavo, vedevo, volevo vedere" trasmette alla frase una potenza visiva. Le immagini plastiche dipingono la lotta, la fanno reale agli occhi dell'autore e del lettore.

"Veder (. . ) i compagni andare, sapendo sul monte": Nella prima edizione c'è, a questo punto, una nota: "sapendo quale destino li attendesse" Nell'edizione posteriore fu giustamente soppressa perché molto più efficace è far sentire al lettore il messaggio senza spiegarlo.

"E volevo imitarli e seguirli, dal soglio dell'opere prese altri monti vedere, altre schiere avverse, altro fuggente paese": nella edizione di Einaudi la frase è modificata. Cosí è la prima stesura: "E volevo imitarli e seguirli, dal soglio dell'opere prese altri monti vedere, altre schiere disperse, altro mare, altro paese" Il termine mare viene spiegato da una nota del commentatore: "Amore per le nuove terre prese al nemico, o da prendere. Il Ns. vedeva delirando una vincente marcia in avanti, la costruiva per fantasia con le immagini degli assalti e delle battaglia a cui aveva realmente partecipato."

Dopo questa nota c'è ancora, nella prima edizione, tutta una spiegazione della "fantasia bellicosa" dell'autore, e un invito a releggere le parole del capitolo precedente, che trascriviamo perché si possa capire meglio quanto fu sincero Gadda nel suo entusiasmo patriottico:

"Sono un profittatore di guerra: perché ne ho cavato giorni e ore ancor vivi nel ricordo e, dico per dire, desiderati nel sogno. Certe festicciuole da ballo che mecenatizzarono la mia adolescenza piena di umiliazioni; certe sonatine di pianoforte che dovevano introdurmi nella felicità musogonica di borghesia, se il Padre Eterno me lo permette, vorrei fare a meno di ringraziarlo del suo buon cuore. Di certe ore di guerra invece non dirò lo ringrazio, è bestemmia, diró solo che le ho vissute con orgoglio e con gioia, o almeno con la sicurezza allucinata del sonnambulo." (16)

La guerre e la prigionia si fissarono, nel cuore e nella memoria di Gadda, indelebilmente. Sull'onda di questa emozione egli inizia la strada amara della propria esistenza; il dolore e la delusione, la frustrazione di non aver potuto fare abbastanza per la Patria, sono alcune delle basi su cui si proietterà il suo doloroso cammino e tutto il suo lavoro di scrittore. Nelle prime pagine di Il Castello di Udine, profeticamente, predice quello che sarebbe stato il suo destino:

(16) - Gadda C.E., "Dal Castello di Udine verso i monti", in Il Castello di Udine, op. cit. p. 50. 
"Cosí seguiterò il mio cammino solitario. Seguiterò a pagare e servire la necessità, conterò avaramente il poco denaro, loderò la plastile carne delle infarinate bagasce; appetirò cose non lecite; altre sognerò non possibili; e una grata sarà il termine de' pochi miei passi. E leggeró i libri sapientissimi delli scrittori, infino a che, sopra alla mia trapassata sapienza, vi crescerà l'erba.

I pensieri piú belli si dissolveranno, ogni volere, ogni gioia, ogni i piú ardente e tenero senso e memoria; e forse l'amore istesso della mia terra! Come avviene che di là, dietro dal monte, la rosea nube in cenere si discolori. $\mathrm{E}$ in sul muro, che chiude il Campo, si leggerà, mal visibile, un segno: un segno inscritto col sangue.

Crescerà ne' vecchi muri l'urtica: e l'erba di sopra la lassitudine mia.

E l'erba, che serè cresciuta, la mangerà il cavallo, che campato sarà. (17)

Un commento del Dott. Averrois, che è, come abbiamo visto, Gadda che spiega Gadda, indica, con amarezza, il senso recondito di quest'ultima frase: "qui il cavallo è la saluberrima stupidità, superstite e pascolante sopra la vana fatica del pensiero"

(17) - Gadda C.E., "Tendo al mio fine", in Il Castello di Udine, op. cit. p. 16 . 